\title{
Zur Frage der nervösen Beeinflussung des Purinstoffwechsels.
}

$$
\text { Von }
$$

\author{
K. Dresel und H. Ullmann.
}

(Aus der II. Med. Iniv.-Klinik der Charité, Berlin [Direktor: Geh. Med.-Rat Prof. Dr. F. Kraus].)

(Eingegangen am 18. Juni 19\%1.)

Ǔber die nervösen Bahnen, welche den Purinstoffwechsel beeinflussen, sind unsere Kenntnisse noch durchaus mangelhaft. Während andere Gebiete des Stoffwechsels, wie der Zucker- und Wärmehaushalt in dieser Beziehung eingehender erforscht sind, finden sich keinerlei Mitteilungen, die über die nervöse Verbindung zwischen Zentralnervensystem und Erfolgsorgan hinsichtlich der Purinausscheidung irgend etwas aussagen..

Zur Untersuchung dieser Frage schien uns die Wirkung des Coffeins und Diuretins auf den Purinstoffwechsel vor und nach der Durchschneidung von Nervenbahnen von Wichtigkeit.

Hess und $\mathrm{Sch} \mathrm{moll}^{1}$ ) hatten gefunden, daß nach T'egenuß bei sonst gleicher Ernährungsweise die Harnsäureausscheidung vermehrt ist. Sie führten diese Erscheinung mit Rücksicht auf die von Gottlieb und Bodcynski2) gefundene Tatsache des Abbaues dieser Verbindungen auf eine Entmethylierung zurück. Später glaubte Schittenhel m ${ }^{3}$ ) bei Versuchen an Hunden zeigen zu können, daß verfütterte Methylpurine z. T. entmethyliert und zur Bildung von Harnsäure bzw. Allantoin verwandt werden. Levinthal ${ }^{4}$ ) sah nach subcutaner Injektion von Coffein natr. salicyl. einen Anstieg besonders der Purinbasen, aber auch der Harnsäuren im Harn gleichzeitig mit einer Vermehrung der Stickstoff- und Wasserausscheidung. Er führte die Harnsäurevermehrung lediglich auf die diuretische Wirkung des Coffeins zurück. Min kowski5) vermißte ebenso wie $\mathrm{Kr}$ üger und $\mathrm{Schmidt^{6 }}$ ) eine Zunahme des Harnsäuregehaltes im Urin nach Einführung selbst großer Coffeindosen.

Von einem ganz neuen Gesichtspunkte aus betrachtete Brugsch ${ }^{7}$ ) clie Harnsäuremehrausscheidung nach Coffeingaben, die er in gemeinsamen Versuchen mit Ka vashima des öfteren gefunden hatte. Zum Verständnis der Schlußfolgerungen, die Brugsch zog, müssen wir kurz auf die Befunde eingehen, die das Studium der Einwirkung des ('offeins und Diuretins auf den Zuckerstoffwechsel ergeben hatte. 
k. Dresel und H. Ullmann: Zur Frage der nervüsen Becinflussung usw. 215

$\mathrm{J}$ k kob $\mathrm{y}^{8}$ ) fand im Jahre 1895 , daß nach intravenöser, subcutaner oder stomachaler Zufuhr von Coffein oder Diuretin Polyurie und Glykosurie auftreten. Er nahm an, daß es sich um einen Nierendiabetes handle. Fast gleichzeitig beschrieb $\mathrm{Neumann}{ }^{9}$ ) das Erscheinen von Zucker im Urin eines Herzkranken nach Diuretinmedikation und glaubte, da $B$ zum Zustandekommen der Glykosurie vorher eine Hyperglykämie bestanden haben müsse. Diese Hyperglykämie wurde von Richter ${ }^{10}$ ) nachgewiesen, der auf Grund dieses Befundes die Hypothese, daß es sich um einen Nierendiabetes handele, ablehnen konnte. R. glaubte, daß durch das Diuretin der Zucker aus der Leber ausgeschwemmt wird, da die Lebern derartig behandelter Tiere nur wenig Glykogen enthielten (s. a. Rose $\mathrm{e}^{11}$ ). Erst Polla $\mathbf{k}^{12}$ ) stellte fest, daß Coffein und Diuretin nach Splanchnicusdurchschneidung nicht mehr glykosurisch wirken, da $B$ also der Angriffspunkt dieser Gifte nicht in der Leber, sondern

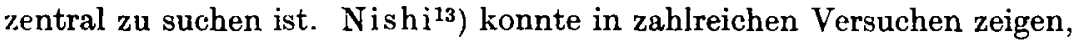
daß der zentrale Reiz, der durch den Splanchnicus verläuft, nicht direkt zur Leber geht, sondern zur Nebenniere. Dementsprechend sah $\mathrm{F} \mathrm{uji}^{\mathbf{1 4}}$ ), daß nach Diuretininjektionen der Adrenalingehalt der Nebennieren abnimmt. Wurde auf einer Seite der Splanchnicus durchschnitten, so war der Adrenalingehalt in der Nebenniere dieser Seite normal.

Somit war festgestellt, daß der Mechanismus der Zuckerstich- und ('offein-Diuretinglykosurie der gleiche ist, da $\mathrm{Kah}^{15}$ ) dieselben Befunde an den Nebennieren nach dem Zuckerstich erhoben hatte.

Unter Berücksichtigung dieser Tatsache glaubte Brugsch (l. c.) annehmen zu dürfen, dal3 auch die Vermehrung der Harnsäureausscheidung nach Coffein auf seine zentrale Wirkung zurückzuführen ist, daß es nämlich nervöse Zentren reize, die für die Purinausscheidung verantwortlich gemacht werden müssen. Brugsch vermutete also ein dem Piqûrezentrum ähnliches Harnsäurezentrum im Zentralnervensystem, das von Einflu $\beta$ auf den Regulationsmechanismus des Purinstoffwechsels in der Leber ist.

Auf seine Veranlassung hat dann Michaelis ${ }^{16}$ ) bei Piqûreversuchen an Kaninchen gefunden, daß bei gelungenem Zuckerstich auch ein starker Anstieg der Allantoinausscheidung und eine Änderung in der gesamten N-Ausscheidung erfolgt. Michaelis schloß hieraus auf ein Zentrum für den Purinstoffwechsel in der Medullar.oblongata, das mit rlem bei der Piqûre getroffenen Zuckerzentrum identisch oder diesem doch benachbart ist. Er bestätigte damit Brugschs Vermutung ,die Piqûre ist wahrscheinlich mehr als ein Zuckerstich". Michaelis glaubte, daß dieser sog. Harnsäurestich ähnlich wie die Piqûre eine Reizung sympathischer Bahnen zur Folge habe, die auf dem Wege über clen Grenzstrang eine Harnsäure- bzw. Allantoinmobilisierung in der Leber bewirke. 
Vom Zuckerstich ist jetzt durch die Untersuchungen von Brugsch, Dresel und Lew $\mathrm{y}^{17}$ ) bekannt, daß er sympathische Zellen im vegetativen Oblongatakern (dorsaler Vaguskern) trifft, von denen Bahnen durch Halsmark, Grenzstrang und Splanchnicus zu den Nebennieren ziehen, durch deren Reizung eine Adrenalinausschüttung und damit eine Zuckermobilisation in der Leber bewirkt wird.

Es handelte sich nun bei unseren Untersuchungen um die Frage, ob der Reiz zur Harnsäureausschwemmung auf denselben eben bezeichneten Bahnen verläuft, wie der Reiz zur Zuckerausscheidung. Zur Klärung dieser Frage gingen wir in der Weise vor, wie es Pollack (l. c.) und Nishi (l.c.) bezüglich der Zuckerausscheidung getan hatten. Da wir Kaninchen als Versuchstiere benutzten, mußte zunächst festgestellt werden, ob Coffein bzw. Diuretin den Purinstoffwechsel dieser Tiere in gleicher Weise beeinflußt, wie es Schittenhelm (l.c.) bei Versuchen am Hund nachgewiesen hatte. In der Tat bewirkte Coffein wie Diuretin regelmäßig einen Anstieg des Allantointageswertes, wie aus den folgenden Tabellen hervorgeht:

Kaninchen VII.

\begin{tabular}{|c|c|c|c|c|c|c|c|}
\hline & Menge & $\begin{array}{c}\text { Spez. } \\
\text { Gewicht }\end{array}$ & Chlor & $\mathrm{N}$ & Allautoin & Zucker & Bemerkungen \\
\hline 8. XII. 1920 & 390 & 1007 & 0,407 & 0,655 & Tageswert & neg. & \\
\hline 9. XII. 1920 & 355 & 1006 & 0,631 & 0,673 & oerecnnet & ", & \\
\hline 10. XII. 1920 & 375 & 1006 & 0,333 & 0,684 & 0,051 g & , & \\
\hline 11. XII. 1920 & 305 & 1009 & 0,380 & 0,964 & Tageswert & , & Tägliche Injek- \\
\hline 12. XII. 1920 & 140 & 1019 & 0,146 & 1,156 & aus 3 Tag. & ,. & $\begin{array}{l}\text { thon von Dure- } \\
\text { tin. } \bar{c} \text { cem einer }\end{array}$ \\
\hline 13. XII. 1920 & 330 & 1008 & 0,117 & $?$ & $0,270 \mathrm{~g}$ & ; & 10 proz. Lösusty. \\
\hline 14. XII. 1920 & 150 & 1014 & 0,106 & 0,441 & Tageswert & , & \\
\hline I5. XII. 1920 & 385 & 1008 & 0,403 & 0,919 & Derecnnet & , & \\
\hline 16. XII. 1920 & 395 & 1007 & 0,413 & 0,804 & $\mathbf{0 , 0 6 5} \mathrm{g}$ & ," & \\
\hline
\end{tabular}

Kaninchen XII.

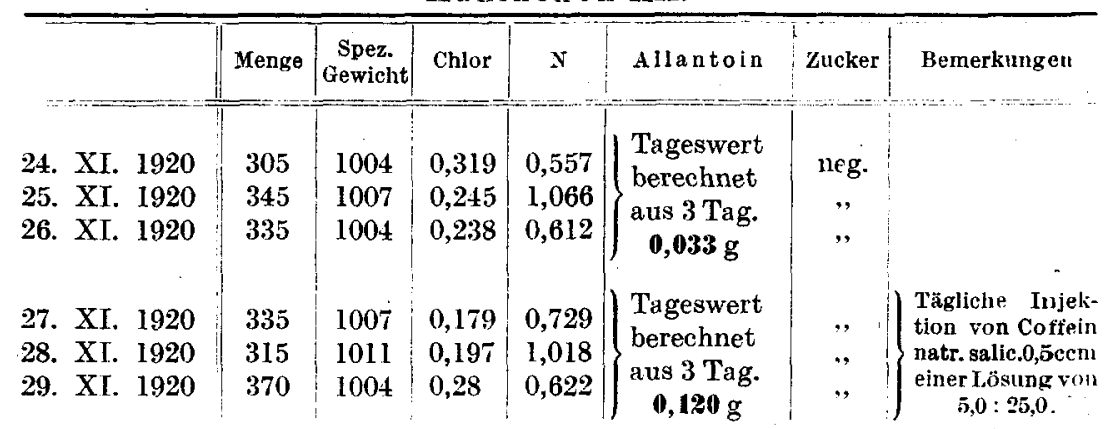


Kaninchen XXII.

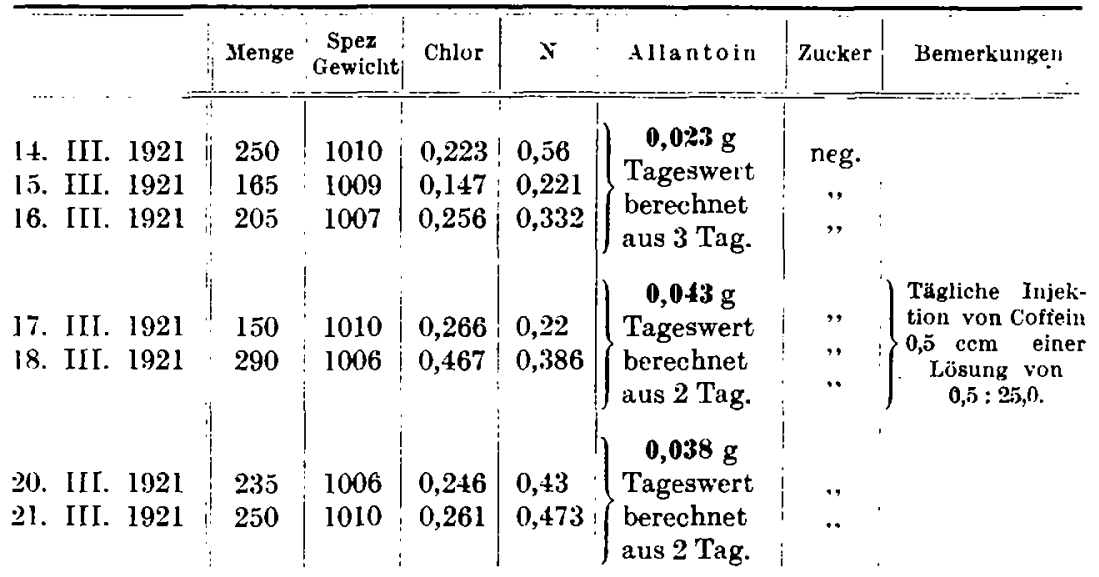

Die Mehrausscheidung war also ganz beträchtlich und betrug in einigen Fällen sogar das $4-5$ fache der Norm. Nach Aussetzen der pharnakologischen Einwirkung fiel die Tagesmenge wieder auf den Ausgangswert ab. (Daß kein Zucker im Urin auftrat, ist nicht verwunderlich, da dieser im Gegensatz zur Hyperglykämie nur bei besonderer Fütterung teohachtet wird.)

Wenn wir nur das Allantoin bestimmt haben und nicht auch die Harnsäure, so gingen wir ebenso wie Schittenhelm von der Tatsache aus [Wiechowski ${ }^{18}$ )], daß das Allantoin das Hauptendprodukt des Purinstoffwechsels bei den Säugetieren mit Ausnahme des Menschen und der anthropoiden Affen ist, also alle Harnsäure, die den Organismus passiert, quantitativ in Allantoin übergeht. Auch hatte Schit tenhelm und Seisser ${ }^{19}$ ) gefunden, daß von intravenös injiziertem Allantoin $100 \%$ im Harn als Purin-N ausgeschieden werden. Zur Bestimmung des Allantoins benutzten wir in den ersten Versuchen die Methode von Wiechowski20), nach der bisher allgemein gearbeitet wurde. Die späteren Untersuchungen sind nach der neuen Methode von Wiechowski ausgeführt, die sich ausschließlich im Neubauer - Huppert ${ }^{21}$ ) findet und die anscheinend bisher noch kaum bekannt geworden ist. Durch eine Reihe von Verbesserungen technischer Natur gestattet sie ein erheblich bequemeres und schnelleres Arbeiten, ohne dadurch an Genauigkeit zu verlieren. Da die Benutzung des heute sehr kostspieligen Bleicarbonats fortfällt und nur geringe Mengen Bleioxyd gebraucht werden, sollte diese Methode in Zukunft allgemein angewandt werden.

Es handelte sich nun darum, festzustellen, ob die Coffein- und Diuretinwirkung auf den Purinstoff wechsel auch bei splanchnicotomierten Tieren zustande kommt, wie dies z. B. beim A ro n soh n- und - S a c h s schen 
Wärmestich der Fall ist, der wie Schultze ${ }^{22}$ ) gezeigt hat, auch nach Splanchnicusdurchschneidung von Erfolg begleitet ist.

Den Kaninchen wurde nach der Methode von Schultze ${ }^{22}$ ) der Splanchnicus beiderseits kurz nach dem Durchtritt durch das Zwerchfell durchtrennt. Daß die Splanchnici in der Tat durchschnitten waren. wurde dadurch erwiesen, daß nach Coffein- bzw. Diuretingaben nunmehr keine Veränderung des Blutzuckers auftrat (siehe z. B. fol-gende Versuche).

Kaninchen XII. Splanchnicotomiert am 21. I. 1921.

Am 4. II. 1921 morgens Blutzucker . . . . . . . . . . . . . 0,110\% 1 Stunde nach Injektion von $5 \mathrm{ccm}$ einer 10 proz. Diuretinlösung . . $0,087 \%$

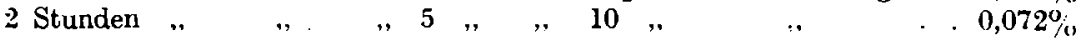

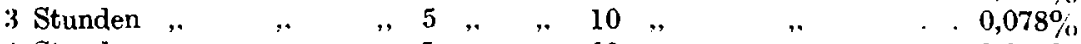

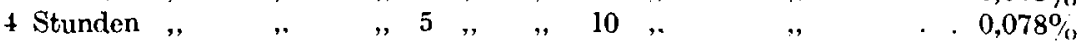

Kaninchen XIX. Splanchnicotomiert am 4. III. 1921.

Am 16. III. morgens Blutzucker . . . . . . . . . . . . . 0,084\% 1 Stunde nach Injektion von $0,5 \mathrm{ccm}$ Coffein einer Lösung von $5,0: 25,0 \quad 0,093 \%$,

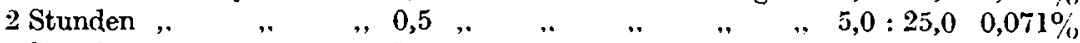

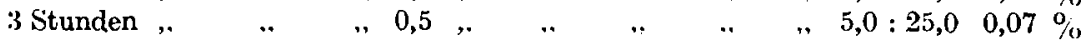

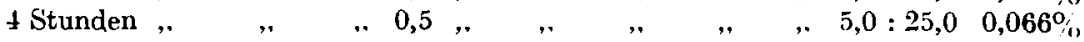

Es wurde abgewartet, bis die Tiere sich völlig von dem operativen Eingriff erholt hatten, was immer einige Tage dauerte, da zunächst die Freßlust stark abnahm. Wiederholt mußte physiologische Koch. salzlösung injiziert werden, um die Diurese in Gang zu bringen. Hatte' sich die Urinausscheidung wieder gleichmäßig eingestellt, so wurden die Tiere in derselben Weise wie bei den vorher besprochenen Versuchen an normalen Tieren mit Coffein bzw. Diuretin behandelt.

Kaninchen XIX.

Am 4. III. 1921 wurde der Splanchnicus beiderseits durchtrennt.

Am 5. IIl. 1921 Injektion von $150 \mathrm{ccm}$ physiol. NaCl-Lösung.

\begin{tabular}{|c|c|c|c|c|c|c|c|}
\hline & Menge & $\begin{array}{l}\text { Spez. } \\
\text { Gewicht }\end{array}$ & Chlor & $\mathrm{x}$ & Allantoin & Zucker & Bemerkungen \\
\hline 13. III. 1921 & 350 & 1007 & $02+8$ & 784 & $0,016 \mathrm{~g}$ & 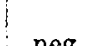 & \\
\hline 14. III. 1921 & 310 & 1010 & 0.275 & 0,586 & Tageswert & . & \\
\hline 15. [II. 1921 & 375 & 1007 & 0,267 & 0,632 & $\begin{array}{l}\text { berechnet } \\
\text { aus } 3 \text { Tag. }\end{array}$ & ". & \\
\hline $\begin{array}{l}\text { 16. UII. } 1921 \\
\text { 17. III. } 1921\end{array}$ & $\begin{array}{r}250 \\
60\end{array}$ & $\begin{array}{l}1010 \\
1019\end{array}$ & $\begin{array}{l}0,261 \\
0,085\end{array}$ & $\begin{array}{l}0,823 \\
0,445\end{array}$ & $\begin{array}{l}\quad 0,019 \mathrm{~g} \\
\text { Tageswert } \\
\text { berechnet } \\
\text { aus } 2 \text { Tag. }\end{array}$ & . & $\begin{array}{l}\text { Tagliche Injek- } \\
\text { tion von } 0,5 \mathrm{ccm} \\
\text { Coffein einer } \mathrm{L} \text { - }- \\
\text { sung von } \\
\mathbf{5 , 0}: \mathbf{2 5 , 0},\end{array}$ \\
\hline
\end{tabular}

Am 18. III. früh 11 thr ging das Versuchstier ein.

Die geringe Differenz des dllantoinwertes liegt innerhalb der Fehlergrenze der Bestimmung. 
Es zeigte sich, daß jetzt eine Allantoinmehrausscheidung nicht mehr stattfand, sondern daß in einigen Fällen sogar ein Rückgang des Allantoingehaltes in dem nach den Injektionen ausgeschiedenen Urin zu heobachten war (siehe die Tabellen Kaninchen XIX und XII).

Kaninchen XII.

Anı 21. I. 1921 wurde der Splanchnicus beiderseits durchtrennt.

Am 27. I. 1921 Injektion von $150 \mathrm{ccm}$ physiol. NaCl-Lösung.

\begin{tabular}{|c|c|c|c|c|c|c|c|}
\hline & Menge & $\left|\begin{array}{c}\text { Spez. } \\
\text { Gewicht }\end{array}\right|$ & Chlor & N & Allantoin & Zucker & Bemerkungen \\
\hline I. II. & 240 & 1010 & 0,298 & 0,145 & $0,043 \mathrm{~g}$ & neg. & . \\
\hline$\because$ II. & 260 & 1008 & 0,230 & 0,528 & Tageswert berech- & , & \\
\hline 3. II. & 190 & 1008 & 0,198 & 0,345 & net aus 3 Tagen - & , & \\
\hline t. II. & 260 & 1010 & 0,184 & 0,510 & $0,0 \approx 7 \mathrm{~g}$ & ,• & (1) Tägliche Injek- \\
\hline స. II. & 250 & 1008 & 0,222 & 0,525 & Tageswert berech- & , & $\left\{\begin{array}{l}\text { ton vol b cem } \\
\text { Diuretin einer }\end{array}\right.$ \\
\hline 6. II. & 200 & 1010 & 0,106 & 0,364 & net aus 3 Tagen & :. & 10 proz. Lösung. \\
\hline ‘. II & 150 & 1007 & 0,080 & 0,336 & $0,048 g$ & , & \\
\hline 8. II. & 215 & 1007 & 0,306 & 0,408 & Tageswert berech- & $\because \cdot$ & \\
\hline 9. II. & 190 & 1010 & 0,303 & 0,279 & net aus 3 'Tagen & ", & \\
\hline 16. II. & 220 & 1007 & 0,156 & 0.385 & $0,033 \mathrm{~g}$ & ,* & \\
\hline 17. II. & 200 & 1008 & 0,355 & 0,378 & Tigeswert berech. & $"$ & \\
\hline 18. II. & 220 & 1006 & 0,312 & 0,339 & net aus 3 Tagen & .. & \\
\hline 19. II. & 180 & 1010 & 0,255 & 0,554 & $0,006 \mathrm{~g}$ & .. & Tägliche Injek- \\
\hline 20. II. & 95 & 1021 & 0,255 & 0,497 & Tageswert berech. & .. & $\begin{array}{l}\text { tion vou } 5 \text { ccill } \\
\text { Diuretin einer }\end{array}$ \\
\hline 2l. II. & 70 & $102: 3$ & 0,124 & 0,524 & net aus 3 Tagen & . & 10 proz. Lösuny \\
\hline 22 . II. & 120 & 1020 & 0,195 & 0,924 & $0,0 z 3 \mathrm{~g}$ & . & \\
\hline 23. II. & 290 & 1009 & 0,154 & 0,934 & 'Tageswert berech- & , & \\
\hline $24 . \mathrm{II}$ & 360 & 1008 & 0,574 & 0,919 & net aus 3 Tagen & ", & \\
\hline
\end{tabular}

Wir sehen hieraus, daß in der Tat der Coffein-Diuretinreiz zur Harnsäure-Allantoinaussch wemmung vom Zentralnervensystem aus über die Splanchnici verläuft, ebenso wie es für die Piqûre- und Coffeinglykosurie früher von Pollack und $\mathrm{Nishi}$ bewiesen worden ist.

Es fragt sich nun, ob dieser Reiz durch den Splanchnicus direkt zur Leber verläuft, oder ob auch hier die Nebenniere eine Rolle spielt, wie es für den Zuckerstich wahrscheinlich gemacht ist.

Für die letztere Annahme scheinen uns folgende Angaben zu sprechen. Falta ${ }^{23}$ ) fand, daß Adrenalin beim Hunde eine ganz erhebliche Mehrausscheidung von Allantoin bedingt. Ferner konnte Rosenberg ${ }^{24}$ ), ${ }^{25}$ ) an unserer Klinik zeigen, daß ein Zusatz von Adrenalin zur Durchblutungsflüssigkeit eine Vermehrung der ausgeschiedenen Purine zur Folge hat.

Diese Angaben zeigen eine auffallende Utbereinstimmung mit den Verhältnissen beim Zuckerstoffwechsel, da seit den Untersuchungen 
von $\mathrm{F} . \mathrm{Blum} \mathrm{m}^{26}$ ) die Zuckerausscheidung nach Adrenalin bekannt ist und da der Adrenalinzusatz zur Durchblutungsflüssigkeit der künstlich durchbluteten Leber eine Mobilisierung von .Traubenzucker bewirkt [Pechstein ${ }^{27}$ ), Dresel und Peiper $\left.{ }^{28}\right)$ ].

Wir müssen demnach angesichts unserer Befunde annehmen, daß3 zum mindesten für die zentrale Coffein-Diuretinwirkung der gleiche Mechanismus sowohl für den Zucker- wie für den Purinstoffwechsel Geltung hat, daß nämlich durch die auf den sympathischen Bahnen vermittelte'Adrenalinaussch wemmung sowohl der Zucker wie die Purine der Leber mobilisiert werden. Inwieweit trotzdem noch im Zentralnervensystem besondere Zentren für den Purinstoffwechsel existieren. läßt sich nach unseren Lntersuchungen bisher nicht entscheiden.

Die angeführten Befunde erlauben uns aber des weiteren Schlüssé zu ziehen hinsichtlich der medikamentösen Beeinflussung der Harnsäureausscheidung. In den diesbezüglichen Versuchen spielte das Coffein lange eine geringe Rolle. Siehe die ausführlichen Arbeiten von Horba czewski ${ }^{29}$ ), Bohland ${ }^{30}$ ), Ulrici ${ }^{31}$ ), Wiechowski ${ }^{32}$ ), Abl ${ }^{34}$ ) und Ne u bauer-H u p pert ${ }^{21}$ ), in denen nichts über die Coffeinwirkung bezüglich des Purinstoff wechsels erwähnt ist.

$\left(\mathrm{Abl}^{33}\right)^{34}$ ) hat die Hypothese aufgestellt, daß die sog. exogene Harnsäureausscheidung nicht direkt durch die vermehrte Zufuhr von Nucleinen in der Nahrung hervorgerufen werde, sondern clurch die infolge Fleischaufnahme angeregte Mehrtätigkeit der Verdauungsdruisen. Hierzu kam er durch die Befunde, die er mit einer Reihe von Medikamenten hinsichtlich der Beeinflussung des endogenen Harnsäurewertes erzielt hatte. Er glaubte einen Parallelismus zu finden zwischen der Durchblutung cles Pfortadergebietes und der Größe der Harnsäureausschwemmung.

Von diesem Gesichtspunkte aus ist die Beeinflussung des Purinstoff wechsels durch Coffein ebenfalls von Bedeutung. Wenn auch in Fermentversuchen an tierischen Organen eine Entmethylisierung des Methylpurins Coffein als nicht unwahrscheinlich bezeichnet wird, so ist doch dieser Abbau, wie schon $\mathrm{Br}$ ugsh vermutet hat und wie auch unsere Versuche zeigen, im Stoffwechsel des tierischen Körpers nicht anzunehmen.

Die Tatsache, daß Coffein beim Menschen nicht regelmäßig eint• Mehrausscheidung von Harnsäure hervorruft, hatte Brugsch auf das Vorhandensein eines mehr oder minder großen Purindepots in der Leber zurückgeführt, was auch mit unserer Auffassung übereinstimmen würde, daß wie beim Zuckerstoffwechsel das Coffein durch Ausschwem. mung von Adrenalin die Purine in der Leber mobilisiert.

Außer dieser indirekten Wirkung auf die Leber hat aber das Coffein einen gefäßverengernden Einfluß auf die Gefäße des: 
Siplanchnicusgebietes. Daß trotzdem durch das Coffein eine Vermehrung der Harnsäureausfuhr bedingt wird, scheint der Ablschen Auffassung zu widersprechen. Dieser Widerspruch klärt sich aber durch die Tatsache auf, daß nach der Splanchnicusdurchschneidung eine Herabsetzung des Harnsäurewertes nach Coffeingaben statt einer Erhöhung von uns gefunden wurde. Somit verdeckt die Purinausschwemmung aus der Leber bei erhaltenem Splanchnicus diese Depression des endogenen Harnsäurewertes. Wenn hiermit auch nichts über die Richtigkeit der Ablschen Anschauung die endogene und exogene Harnsäureausscheidung betreffend gesagt werden soll, so scheint uns unser Vorgehen doch als aussichtsreiche Methode zur weiteren Prüfung der pharmakologischen Beeinflussung des Purinstoffwechsels.

\section{Literaturverzeichnis.}

1) Hess und Schmoll, ther die Beziehungen der Eiweiß- und Paranucleinsubstanzen der Nahrung zur Alloxurkörperausscheidung im Harn. Arch. f. exp. Pathol. u. Pharmakol. 3\%, 243. 1896. - ") Gottlieb und Bodeynski, Úber Methylxanthin, ein Stoffwechselprodukt von Theobromin und Coffein. Arch. $f$. exp. Pathol. u. Pharmakol. 36, 1895 - 3) Sch i t te nh el m, Zur Frage der harnsäurevermehrenden Wirkung von Kaffee und Tee und ihrer Bedeutung in der Gichttherapie. Therap. Monatshefte 24, 113. 1910. - 4) Levinthal, Zum Abbau des Xanthins und Coffeins im Organismus des Menschen. Zeitschr. f. physiol. Chemie 87, 259. 1912. - ${ }^{5}$ ) v. Minkowski, Die Gicht in Nothnagels Handbuch d. Pathol. u. Therap. Wien 1903 . $-{ }^{6}$ ) Kr üger und Sch midt, zitiert nach Minkowski. - ¿) Brugsch, Probleme des Nucleinstoffwechsels im Hinblick auf die Lehre der Gicht. Med. Klinik 1913, Nr. 1, S. 7. ${ }^{8}$ ) J a co b y, Ùber künstlichen Nierendiabetes. Aroh. f. exp. Pathol. u. Pharmakol. 35, 213. 1895. - ${ }^{9}$ ) Neu. mann, Glykosurie bei einem Herzfehler. Arch. f. exp. Pathol. u. Pharmakol. 36, 72. 1895. - 10) Richter, Diuretica und Glykosurie nebst Versuchen über Glykogenbildung. Zeitscbr. f. klin. Med. 35, 463. 1898. - 11) Rose, Der Blutzuckergehalt des Kaninchens, seine Erhöhung durch den Aderlaß, durch die Eröffnung der Bauchhöhle und durch die Nierenausschaltung und sein Verhalten im Diuretindiabetes. Arch. f. exp. Pathol. u. Pharmakol. 50, 15. 1903. 12) Pollack, Kritisches und Experimentelles zur Klassifikation der Glykosurien. Arch. f. exp. Pathol. u. Pharmakol. 61, 376. 1909. - ${ }^{13}$ ) Nishi, Über den Mechanismus der Diuretinglykoswrie. Arch. f. exp. Pathol. u. Pharmakol. 61, 401. 1909.14) F u ji, Utber die Veränderungen des Gehaltes der Nebennieren an chromaffiner Substanz bei einigen experimentellen Diabetesformen zentralen Ursprungs. Journ. of exp. med. 1, Nr. 1. 1920. - ${ }^{15}$ ) Kah n, Zuckerstich und Nebennieren. Pflügers Arch. f. d. ges. Physiol. 140, 209. 1911. - ${ }^{\text {16) }}$ Michaelis, Zur Frage des intermediären Purinstoffwechsels, 2. Mitteilung. Uber den Harnsäurestich. Zeitschr. f. exp. Pathol. u. Therap. 14, 255. 1913. - 17) Brugsch, Dresel und Lewy, Beiträge zur Stoffwechselneurologie. 1. Mitteilung: Zur Stoffwechselneurologie der Medulla oblongata. Zeitsebr. f. exp. Pathol. u. Therap. 21. 1920. 18) Wiechowski, Die Bedeutung des Allantoins im Harnsäurestoffwechsel. Beitr. f. chem. Physiol. u. Pathol. 11, 109. 1908. ${ }^{19}$ ) Schit te $\mathrm{nhel} \mathrm{m}$ und Seisser, Über die.Beeinflussung des Stoffwechsels vom Kaninchen und Hund durch Zufubr von Nucleinsäure, Harnsäure und Allantoin. Zeitschr. f. exp. Pathol. u. Therap. g, 116. 1909. - 20) Wiechowski, Die Produkte der fermentativen Harnsäure. 
222 K. Dresel u. H. Ullınını: Zur Frage der nervösen Beeinflussung usw.

zersetzung durch tierische Organe. Beitr. z. chem. Physiol. u. Pathol. 9, 295. 1907. -_ 21) Neubauer - Hu ppert, Analyse des Harns. 2, 11. Aufl. 914. 1913. *2) Schultze, Über den Wärmehaushalt des Kaninchens nach dem Wärmestich. Arch. f. exp. Pathol. u. Pharmakol. 43, 204. 1900. - ${ }^{23}$ ) Falta, Studien über den Purinstoffweehsel. 1. Mitteilung: Der Einfluß des Adrenalins auf die Allantoinausscheidung beim Hunde. Zeitschr. f. exp. Pathol. u. Therap. 15, 356. 1914. 24) Rosenberg, Versuche und Betrachtungen über den Purinstoffwechsel mit einer experimentell kritischen Analyse der Atophanwirkung. Inaug.-Diss. Berlin 1914. - 25) Rosenberg, Zur Frage des intermediären Purinstoffwechsels. 3. Mitteilung: Kritisches über Leberdurchblutungsversuche, Purinumsatz und Atophanwirkung. Zeitschr. f. exp. Pathol. u. Therap. 20. 1919. - ${ }^{28}$ ) Bl u m, Über Nebennierendiabetes. Dtsch. Arch. f. klin. Med. 11. 1901. - 27) Pech. stein, Zur Frage des experimentellen Diabetes. 1. Mitteilung: Zuckermobilisation durch Adrenalin in Leberdurchblutungsversuchen. Zeitschr. f. exp. Pathol. u. Therap. 12. 1913. $-{ }^{28}$ ) Dresel und Peiper, Zur Frage des experimentellen Diabetes, Beeinflussung der Zuckermobilisation durch Adrenalin und Pankreasextrakte in der künstlich durchbluteten Leber. Zeitschr. f. exp. Pathol. u. Therap. 16. 1914. - ${ }^{29}$ ) Horbaczewski, Beiträge zur Kenntnis der Bildung der Harnsäuren und der Xanthinbasen sowie der Entstehung der Leukocyten im Säugetierorganismus. Monatshefte f. Chemie 12, 221 u. 322. 1891. - 30) Bohland. Über den Einfluß einiger Arzneimittel auf die Bildung und Ausscheidung der Harnsäure. Münch. med. Wochenschr. 1899, Nr. 16. - ${ }^{31}$ ) Ulrici, Über pharmakologische Beeinflussung der Harnsäureausscheidung. Inaug.-Diss. Marburg 1901.32) Wiechowski, Beiträge zur Physiologie u. Pharmakologie d. Purinhaushalts der Säugetiere. Münch. med. Wochenschr. 1912, S. 1252. - ${ }^{33}$ ) Abl, Über die Beziehung zwischen Splanchnikotonus und Harnsäureausfubr. Kongr. f. inn. Med. Wiesbaden 1913, S. 187. - ${ }^{34}$ ) Abl, Pharmakologisehe Beeinflussung der Harnsäureausscheidung. Arch. f. exp. Pathol. u. Pharmakol: r4, 119. 1913. 